\title{
Structural analysis and dielectric relaxation mechanism of conducting polymer/volcanic basalt rock composites
}

\author{
Ayşe Evrim BulgurcuoĞlu, Yaşar Karabul, Mehmet Kiliç*, Zeynep Güven Özdemir, \\ Seda ERdönmez, BANu SÜNGÜ MisirlioĞLu, Mustafa OKutan, ORHAN İÇElli \\ Department of Physics, Yildiz Technical University, 34220 İstanbul, Turkey
}

\begin{abstract}
In this work, polypyrrole and polythiophene conducting polymers (CPs) have been synthesized and doped with volcanic basalt rock (VBR) in order to improve their dielectric properties for technological applications. The structure and morphology of the composites with different VBR doping concentrations were characterized by FT-IR and SEM analyses. The best charge storage ability was achieved for maximum VBR doping concentration (50.0 wt.\%) for both CPs. Dielectric relaxation types of the composites were determined as non-Debye type due to non-zero absorption coefficient and observation of semicircles whose centers were below $Z^{\prime}$ axis at the Nyquist plots. It was also ascertained that VBR doping makes the molecular orientation easier than for non-doped samples and reduced energy requirement of molecular orientation. In addition, AC conductivity was totally masked by DC conductivity for all samples at low frequency.
\end{abstract}

Keywords: composite materials; polypyrrole; polythiophene; volcanic basalt rock; dielectric spectroscopy

\section{Introduction}

In recent years, there has been an increasing attention on polymer based biosensors [1], supercapacitors [2] and other electronic and optoelectronic devices $[3,4]$ due to promising properties of conducting polymers (CPs). Among them, polypyrrole (PPy) and polythiophene (PT) are two of the most preferred CPs due to their easy and low cost fabrication, high electrical conductivity and good thermal and environmental stabilities [5]. Furthermore, their electrical conductivity, electrochemical performances and magnetic properties can be developed successfully by doping with various dopants [6] or constructing organic/inorganic composite structures [7-14]. For instance, it has been shown that boric acid can be a good candidate for controlling the electrical conductivity of PPy [6]. Also Bai et al. [7] have concluded that the coating of PT on the surface of $\mathrm{LiFePO}_{4}$ particles leads to a significant increase in electronic conductivity. Shen et al. [12] have shown that PPy coating positively affects the electrical

*E-mail: kilic-m@hotmail.com conductivity of z-type barium ferrite/silica composites. Additionally, PPy has also been suggested as a unique material for the improvement of device applications such as in supercapacitor [8] and sensor structures [9]. It has been clearly reported that CPs have a crucial importance for improving performance of the technological applications. In this study, in the light of their promising characteristics, we aimed to investigate both structural and dielectric properties of PPy and PT polymers. On the other hand, conducting PT and PPy have been utilized as an electromagnetic shielding (EMS) material [15-18]. However, they have some limitations in applications such as poor flexibility, high weight, etc. To overcome these problems, some researchers have tried different polymer based composites [1517]. In this respect, we have chosen basalt as a doping material due to its remarkable advantages, such as thermal stability, EMS property etc.

As is known, basalt is a very widespread volcanic rock that is dark colored and relatively rich in iron and magnesium. Basalt is almost found in nature all around the world [19]. Basalt is chemically rich with oxides of iron, silicon, potassium, sodium, calcium, and magnesium, along with 
traces of alumina. The chemical content may differ based on the geographical location. It is utilized for a wide range of areas, such as building tiles, highway engineering, and industrial constructions. Also the fibers and powders of basalt rocks are widely used for sound insulation, thermal stabilization, heat and radiation shielding [20-22]. Additionally, it is well known that basalt can be used for fabricating heterojunction or composite structures [23]. In this work, we have firstly doped PPy and PT with various concentrations of basalt and focused on the improvement of electrical and dielectric properties of PPy and PT.

In our experiments, the conductive polymer/basalt composites have been prepared by mechanical mixing method. The electrical and dielectric properties of the composites have been investigated by impedance spectroscopy method.

\section{Experimental}

\subsection{Synthesis of conducting polymers and their characterization}

Pyrrole $(\mathrm{CHN})$, thiophene $\left(\mathrm{C}_{4} \mathrm{H}_{4} \mathrm{~S}\right)$, anhydrous iron (III) chloride $\left(\mathrm{FeCl}_{3}\right)$ were supplied by Merck (Germany). Chloroform $\left(\mathrm{CHCl}_{3}\right)$ and methanol $\left(\mathrm{CH}_{3} \mathrm{OH}\right)$ were purchased from Sigma Aldrich.

For the synthesis of polypyrrole (PPy), $0.02 \mathrm{~mol}$ pyrrole was mixed in $70 \mathrm{~mL}$ chloroform under stirring with magnetic stirrer. $0.055 \mathrm{~mol} \mathrm{FeCl}_{3}$ was dissolved in $180 \mathrm{~mL}$ chloroform and added dropwise to the mixture. The solution was allowed to polymerize for $24 \mathrm{~h}$. Then it was filtered and washed in chloroform and methanol, respectively. Black colored PPy sediment was dried in an oven at $60{ }^{\circ} \mathrm{C}$ for $24 \mathrm{~h}$. Similar to the PPy polymerization, polythiophene (PT) was synthesized by polymerization of thiophene using $\mathrm{FeCl}_{3}$ as an oxidizing agent by chemical oxidative polymerization. In order to achieve the molar ratio of $\mathrm{n}_{\text {oxidant }} / \mathrm{n}_{\text {monomer }}=$ 2.5, $0.02 \mathrm{~mol}$ thiophene was mixed with $70 \mathrm{~mL}$ chloroform with a magnetic stirrer for $10 \mathrm{~min}$. In addition, $0.055 \mathrm{~mol} \mathrm{FeCl}_{3}$ was dissolved in $180 \mathrm{~mL}$ chloroform and added to the first solution in dropwise fashion. The mixed solution was stirred for $24 \mathrm{~h}$ at room temperature. Then the solution was filtered and washed in chloroform and methanol. During this process it was observed that the black color of the precipitate became brown. The product was dried in an oven at $60^{\circ} \mathrm{C}$ for $24 \mathrm{~h}$ The chemical oxidative polymerizations of PPy and PT have been shown in Fig. 1a and Fig. 1b, respectively.

(a)

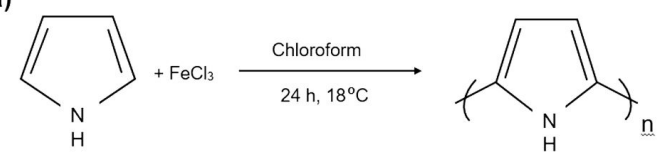

(b)

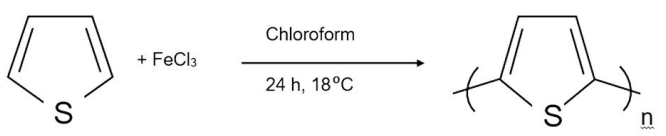

Fig. 1. Chemical oxidative polymerization of (a) PPy, and (b) PT.

Fourier transform infrared (FT-IR) spectra of pure CPs have been recorded with Perkin Elmer Spectrum 100 model spectrometer within the wavenumber range of $4000 \mathrm{~cm}^{-1}$ to $650 \mathrm{~cm}^{-1}$. The FT-IR spectra of PPy and PT have been shown in Fig. 2a and Fig. 2b, respectively.

According to Fig. 2, FT-IR peaks characteristic of PPy and PT are observed. In the PPy spectrum (Fig. 2a), a broad band that appears at around $3381 \mathrm{~cm}^{-1}$ is caused by the $\mathrm{N}-\mathrm{H}$ stretching vibration which proves the existence of pyrrole [24-26]. The peak at $909 \mathrm{~cm}^{-1}$ is assigned to $\mathrm{C}-\mathrm{H}$ outof-plane deformations. The peaks at $1047 \mathrm{~cm}^{-1}$, $1411 \mathrm{~cm}^{-1}$ and $1596 \mathrm{~cm}^{-1}$ are ascribed to $\mathrm{C}-\mathrm{H}$, $\mathrm{C}-\mathrm{N}$ and $\mathrm{C}=\mathrm{C} / \mathrm{C}-\mathrm{C}$ stretching vibrations in pyrrole rings, respectively [24-29]. In the FT-IR spectrum of PT (Fig. 2b), the bands in the range of $600 \mathrm{~cm}^{-1}$ to $1500 \mathrm{~cm}^{-1}$ is the fingerprint region of PT. The peak situated at $656 \mathrm{~cm}^{-1}$ is ascribed to $\mathrm{C}-\mathrm{S}$ stretching of the thiophene ring [30-33]. Also, the peak around $711 \mathrm{~cm}^{-1}$ is attributed to $\mathrm{C}-\mathrm{S}$ bending mode $[33,34]$. The spectrum shows the $\mathrm{C}-\mathrm{H}$ out-of-plane deformation at $772 \mathrm{~cm}^{-1}$ and in-plane $\mathrm{C}-\mathrm{H}$ aromatic bending vibrations at $1084 \mathrm{~cm}^{-1}$ and $1191 \mathrm{~cm}^{-1}$ [30-32]. The peaks at $1385 \mathrm{~cm}^{-1}$ and $1634 \mathrm{~cm}^{-1}$ correspond to 
$\mathrm{C}-\mathrm{C}$ [32] and $\mathrm{C}=\mathrm{C}$ stretching vibrations, respectively. The peak appearing at $3353 \mathrm{~cm}^{-1}$ is attributed to $\mathrm{O}-\mathrm{H}$ stretching vibrations [31].
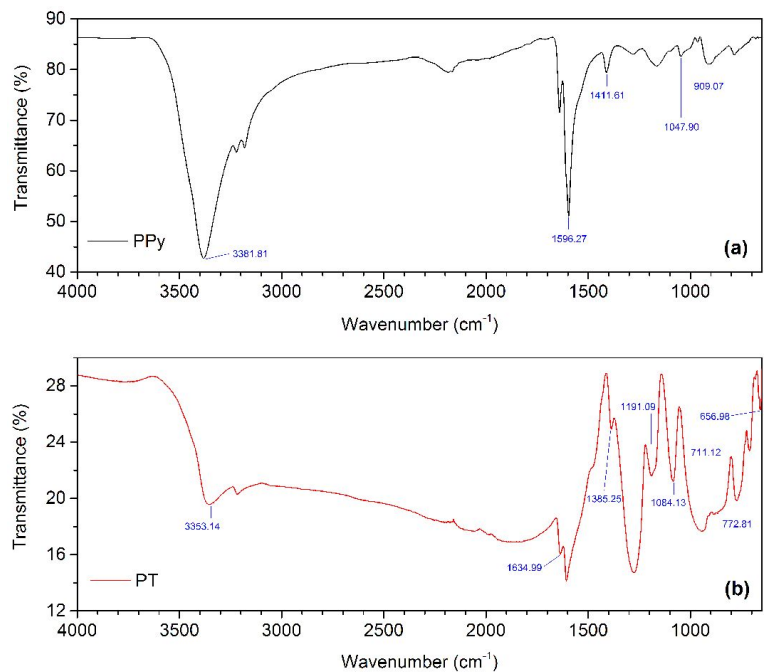

Fig. 2. FT-IR spectra of (a) pure PPy, and (b) pure PT.

\subsection{Preparation and characterization of the samples}

Volcanic basalt rock (VBR) material, which was chosen as the dopant material for CPs, was collected from Van city, Turkey. Chemical analysis of the VBR was taken by X-ray fluorescence (XRF) instrument in our previous work [35]. Operating conditions of the Philips PW-2400 XRF instrument were set at $60 \mathrm{kV}$ and $50 \mathrm{~mA}$. The result of chemical analysis of the sample was given in Table 1.

According to the chemical composition analysis, the sample contains more than $45 \%$ and less than $52 \%$ of $\mathrm{SiO}_{2}$ and less than $5 \%$ of total alkalis $\left(\mathrm{Na}_{2} \mathrm{O}+\mathrm{K}_{2} \mathrm{O}\right)$. Due to this chemical composition of $\mathrm{SiO}_{2}$ and total alkalis, the doping material has been considered as volcanic basalt rock (VBR) [19].

In order to analyze the effect of VBR on the dielectric properties of CPs, different percentages (10.0 wt.\%, 25.0 wt.\%, 50.0 wt.\%) of VBR doping were tried. The CP/VBR mixtures were ground by using IKA A11 basic analytical mill with rotational speed of $10000 \mathrm{rpm}$. The milling was performed in a $250 \mathrm{~mL}$ volume chamber using $30 \mathrm{~mm}$ steel balls. The mixing time per sample was 15 min. After this grinding process, each mixture became a homogeneous powder. For the measurements, each composite was prepared in the form of pellets with $13 \mathrm{~mm}$ diameter and $1.9 \mathrm{~mm}$ to $2.1 \mathrm{~mm}$ thickness by using a hydraulic press with a pressure of ca. $2.76 \times 10^{7} \mathrm{~Pa}$.

The morphology of the samples has been determined by scanning electron microscopy measurements performed using Zeiss-EVO® LS 10 model scanning electron microscope (SEM). The samples were observed under an accelerating voltage of $10.00 \mathrm{kV}, \times 1000$ to $\times 20000$ magnification, and $5 \mathrm{~mm}$ of working distance. The SEM micrographs of the PPy and PT samples have been shown in Fig. 3 and Fig. 4, respectively.

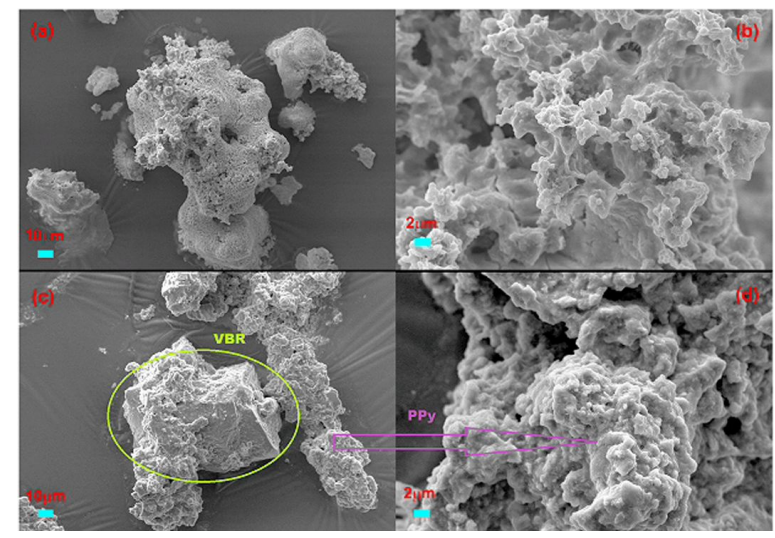

Fig. 3. SEM micrographs of the PPy samples (a), (b) pure, and (c), (d) PPy+(25\%)VBR.

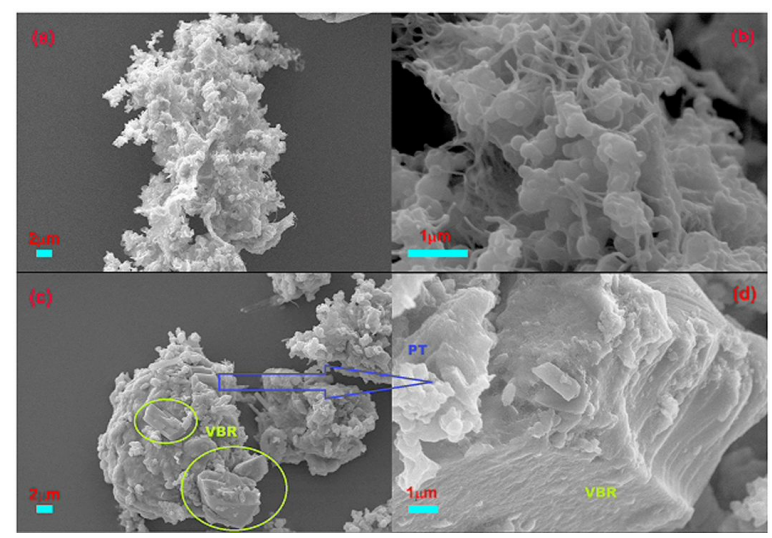

Fig. 4. SEM micrographs of the PT samples (a), (b) pure PT, (c), (d) PT+(25\%)VBR. 
Table 1. Chemical composition of the VBR sample.

\begin{tabular}{llllllllllll}
\hline Comp. & $\mathrm{SiO}_{2}$ & $\mathrm{TiO}_{2}$ & $\mathrm{Al}_{2} \mathrm{O}_{3}$ & $\mathrm{Fe}_{2} \mathrm{O}_{3}$ & $\mathrm{MnO}$ & $\mathrm{MgO}$ & $\mathrm{CaO}$ & $\mathrm{Na}_{2} \mathrm{O}$ & $\mathrm{K}_{2} \mathrm{O}$ & $\mathrm{P}_{2} \mathrm{O}_{5}$ & Total \\
\hline \hline wt.\% & 47.790 & 1.3950 & 16.918 & 10.878 & 0.1630 & 7.6190 & 11.357 & 3.1370 & 0.5190 & 0.2240 & 100 \\
\hline
\end{tabular}

According to the SEM results, it has been observed that VBRs incorporated into the CPs and the dimension of VBR particles are of the order of between $8 \mu \mathrm{m}$ and $50 \mu \mathrm{m}$. It can also be seen that the VBR particles are composed of randomly dispersed almost rectangular parallelepiped shaped forms. This means that VBR has not diffused into the CPs and also has not been dissolved but VBR doping resulted in the formation of a composite structure.

The particle size distribution of VBR has also been determined by Malvern Mastersizer, Hydro 2000MU model as in our previous work. As is seen from Table 2, $93 \%$ of the VBR particles size is smaller than $69.183 \mu \mathrm{m}, 49 \%$ of the VBR particles' size is smaller than $22.909 \mu \mathrm{m}$, and $11 \%$ of the VBR particles' size is smaller than $2.188 \mu \mathrm{m}$.

\section{Results and discussion}

\subsection{Frequency dependent dielectric pa- rameters}

The composites have been placed between two gold electrodes whose surfaces wholly overlapped the faces of the pellets [36]. The dimension of the electrodes was $25 \mathrm{~mm} \times 20 \mathrm{~mm}$. Dielectric measurements were performed by using an HP 4194A impedance analyzer, in the frequency range of $100 \mathrm{~Hz}$ to $15 \mathrm{MHz}$ at room temperature with a high accuracy $(0.17 \%)$ The experimental results were transferred to a computer with GPIB data cable and simultaneously recorded by the computer. In this work, the overall errors of the dielectric measurements were $2.5 \%$.

The frequency dependencies of the real parts of dielectric function of PPy and PT samples have been given in Fig. 5a and Fig. 5b, respectively.

It has been observed that the general behavior of all the $\varepsilon^{\prime}(\omega)$ curves in Fig. 5 indicates a decreasing trend with increasing frequency. On the other hand, VBR doping ratio increases the $\epsilon^{\prime}(\omega)$ values of the CPs at low frequency region except for PT+(10\%) VBR sample. However, regardless of the conducting polymer, the maximum dielectric permittivity value has been achieved for the maximum VBR doping concentration (50\%). As suggested by Panwar et al. [37], the composites with the highest dielectric constant can be used in charge storing devices at low frequency. In this context, it has been deduced that VBR doping enhances the ability of charge storage of the CPs. In addition to this implication, both VBR doping and frequency changes have not affected $\varepsilon^{\prime}(\omega)$ values of the PPy and PT pellets above $4.95 \times 10^{4} \mathrm{~Hz}$ and $14.4 \times 10^{4} \mathrm{~Hz}$, respectively.

The angular frequency dependence of the real component of dielectric function at low frequency region for all samples can be explained by the fact that the electric dipoles in the materials orient themselves along the electric field applied, which corresponds to a polarization process. As is known, when an electromagnetic radiation signal is applied to solids, four types of possible polarization processes (electronic, atomic or ionic, dipolar or orientational and interfacial) can be observed. Each polarization is observed at different frequency region. While electronic polarization occurs at ultraviolet region of electromagnetic spectrum $\left(10^{15} \mathrm{~Hz}\right)$, atomic or ionic polarizations generally appear in far infrared wave frequencies $\left(10^{10} \mathrm{~Hz}\right.$ to $\left.10^{13} \mathrm{~Hz}\right)$. Dipolar, i.e. orientational and interfacial polarizations, are also observed at radio waves $\left(10^{6} \mathrm{~Hz}\right.$ to $\left.10^{3} \mathrm{~Hz}\right)$ and ultra-low frequency waves $\left(10^{3} \mathrm{~Hz}\right.$ to $10^{2} \mathrm{~Hz}$ ), respectively $[38,39]$. Since the angular frequency dependence of $\varepsilon^{\prime}(\omega)$ is observed up to $10^{4} \mathrm{~Hz}$ and $10^{5} \mathrm{~Hz}$ for PPy and PT composites, this behavior of $\mathcal{E}^{\prime}(\omega)$ can be attributed to the existence of dipolar relaxation process in the materials.

Dielectric relaxation type of CPs/VBR composites has also been investigated by Cole-Cole relation given in equation 1 : 
Table 2. Particle size distribution of VBR.

\begin{tabular}{cccccc}
\hline Size $[\mu \mathrm{m}]$ & Volume $[\%]$ & Size $[\mu \mathrm{m}]$ & $\begin{array}{c}\text { Volume } \\
{[\%]}\end{array}$ & Size $[\mu \mathrm{m}]$ & $\begin{array}{c}\text { Volume } \\
{[\%]}\end{array}$ \\
\hline \hline $0.010-0.316$ & 0.00 & 2.512 & 1.38 & 22.909 & 4.32 \\
0.363 & 0.01 & 2.884 & 1.44 & 26.303 & 4.65 \\
0.417 & 0.11 & 3.311 & 1.50 & 30.200 & 4.96 \\
0.479 & 0.21 & 3.802 & 1.58 & 34.674 & 5.22 \\
0.550 & 0.33 & 4.365 & 1.68 & 39.811 & 5.38 \\
0.631 & 0.44 & 5.012 & 1.80 & 45.709 & 5.39 \\
0.724 & 0.56 & 5.754 & 1.95 & 52.481 & 5.19 \\
0.832 & 0.67 & 6.607 & 2.11 & 60.256 & 4.74 \\
0.955 & 0.79 & 7.586 & 2.30 & 69.183 & 4.05 \\
1.096 & 0.89 & 8.710 & 2.50 & 79.433 & 3.17 \\
1.096 & 0.99 & 10.000 & 2.72 & 91.201 & 2.19 \\
1.259 & 1.08 & 11.482 & 2.94 & 104.713 & 1.18 \\
1.445 & 1.16 & 13.183 & 3.19 & 120.226 & 0.23 \\
1.660 & 1.22 & 15.136 & 3.44 & $138.038-1000.000$ & 0.00 \\
1.905 & 1.28 & 17.378 & 3.71 & & \\
2.188 & 1.33 & 19.953 & 4.01 & & \\
\hline
\end{tabular}

Table 3. Absorption coefficient $\alpha$ relaxation time $\tau_{0}$, and other dielectric parameters.

\begin{tabular}{lcccccc}
\hline \multicolumn{1}{c}{ Sample } & $\mathrm{R}^{2}$ & $\alpha$ & $\tau_{0}[\mathrm{~s}]$ & $\varepsilon_{s}$ & $\varepsilon_{\infty}$ & $\Delta \varepsilon$ \\
\hline \hline PPy & 0.99682 & $0.242 \pm 0.12 \times 10^{-1}$ & $28.20 \times 10^{-4} \pm 1.3 \times 10^{-2}$ & $1.3140 \times 10^{4}$ & 24.16 & $0.13115 \times 10^{5}$ \\
PPy $+(10 \%)$ VBR & 0.99994 & $0.150 \pm 9.27 \times 10^{-4}$ & $6.43 \times 10^{-4} \pm 3.21 \times 10^{-5}$ & $2.16803 \times 10^{5}$ & 28.90 & $2.16775 \times 10^{5}$ \\
PPy $+(25 \%)$ VBR & 0.99991 & $0.128 \pm 1.57 \times 10^{-3}$ & $4.25 \times 10^{-4} \pm 1.54 \times 10^{-5}$ & $6.77889 \times 10^{5}$ & 4.87 & $6.77884 \times 10^{5}$ \\
PPy + (50\%) VBR & 0.99998 & $0.126 \pm 2.76 \times 10^{-3}$ & $3.06 \times 10^{-4} \pm 1.05 \times 10^{-5}$ & $2.98169 \times 10^{6}$ & 171.39 & $2.98151 \times 10^{6}$ \\
PT & 0.99947 & $0.223 \pm 1.88 \times 10^{-2}$ & $13.97 \times 10^{-3} \pm 10.90 \times 10^{-3}$ & $6.16332 \times 10^{7}$ & 974.94 & $6.16322 \times 10^{7}$ \\
PT + (10\%) VBR & 0.99883 & $0.178 \pm 4.08 \times 10^{-2}$ & $10.20 \times 10^{-3} \pm 15.15 \times 10^{-2}$ & $4.72632 \times 10^{8}$ & 1913.90 & $4.72630 \times 10^{8}$ \\
PT + (25\%) VBR & 0.99994 & $0.189 \pm 8.72 \times 10^{-4}$ & $4.38 \times 10^{-3} \pm 1.73 \times 10^{-4}$ & $9.14618 \times 10^{8}$ & 1166.80 & $9.14616 \times 10^{8}$ \\
PT + (50\%) VBR & 0.99994 & $0.206 \pm 9.65 \times 10^{-4}$ & $4.16 \times 10^{-3} \pm 1.58 \times 10^{-4}$ & $7.49987 \times 10^{8}$ & 851.52 & $7.49986 \times 10^{8}$ \\
\hline
\end{tabular}

$$
\begin{aligned}
& \varepsilon^{\prime}(\omega)=\varepsilon_{\infty}+ \\
& \left(\varepsilon_{s}-\varepsilon_{\infty}\right) \frac{1+\left(\omega \tau_{0}\right)^{1-\alpha} \sin \frac{1}{2} \alpha \pi}{1+2\left(\omega \tau_{0}\right)^{1-\alpha} \sin \frac{1}{2} \alpha \pi+\left(\omega \tau_{0}\right)^{2(1-\alpha)}}
\end{aligned}
$$

where $\varepsilon_{\infty}$ and $\varepsilon_{\mathrm{s}}$ are the high and low limiting angular frequency dielectric constants. $\tau_{0}$ and $\alpha$ represent relaxation time and absorption coefficient, respectively [40].

In general, absorption coefficient parameter, $\alpha$, varies between zero and one $(0<\alpha \leqslant 1)$. If $\alpha$ equals to zero, it corresponds to standard Debye type relation. The non-Debye type occurs when the value of absorption coefficient varies between $(0,1)$ region [41].

Dispersion curves given in Fig. 5 have been fitted by Origin Lab 8.5 graphic program in accordance with equation 1 . The fitting results have been listed in Table 3.

Since absorption coefficient values of all samples are greater than zero, relaxation mechanism has been considered as non-Debye type. In addition, the dielectric strength $\Delta \varepsilon$ has been calculated from the difference 

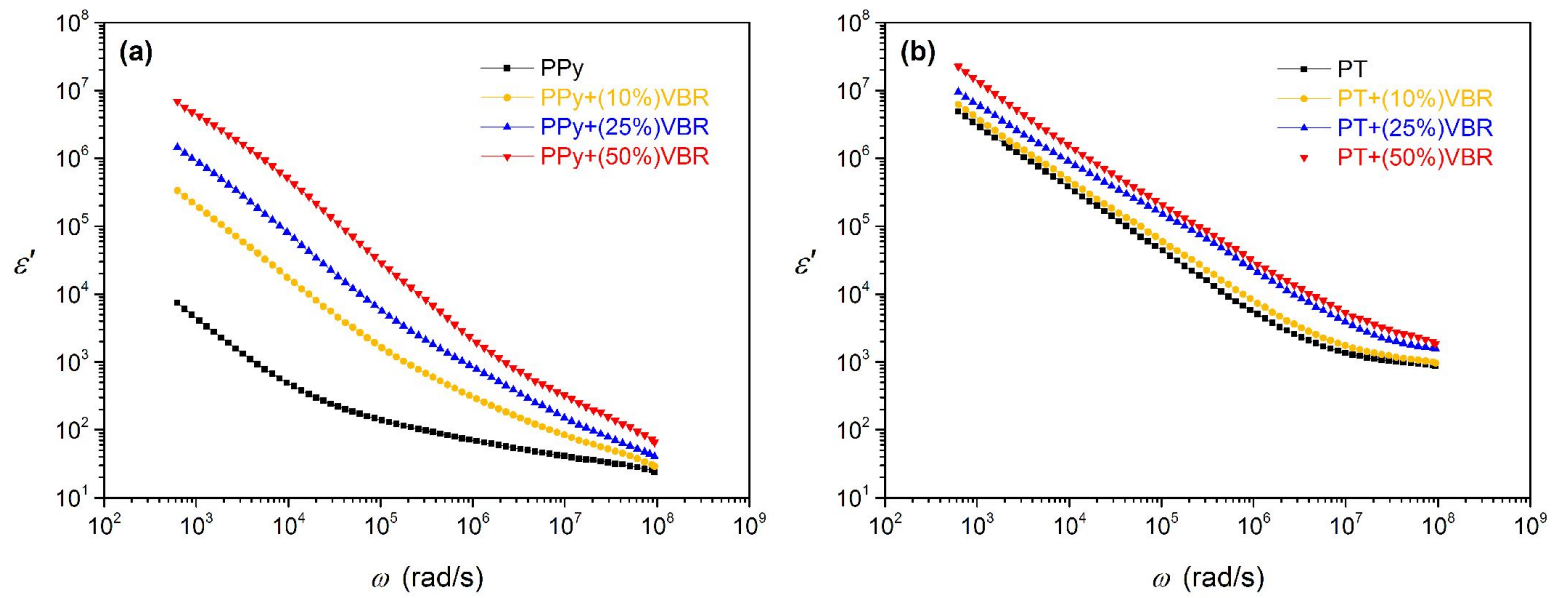

Fig. 5. Angular frequency dependence of $\epsilon^{\prime}$ for (a) pure PPy and PPy/VBR composites, (b) pure PT and PT/VBR composites.

between the limiting low frequency and high frequency dielectric constant and listed in Table 3. As is seen from Table 3, dielectric strength values considerably increase with incremental VBR concentration for both CPs. In this respect, one can deduce that the increase of dielectric strength with VBR doping makes the electric dipole orientation easier relative to non-doped samples and hence, VBR dopant reduces the energy requirement of this orientation.

The variations of the imaginary parts of dielectric function with angular frequency of PPy and PT samples have been given in Fig. 6a and Fig. 6b, respectively. As shown in Fig. 6, the imaginary components of dielectric function, i.e. dielectric loss of both CPs and the related composites decrease with angular frequency and show strong frequency dependence in low frequency region. It has also been observed that the $\varepsilon^{\prime \prime}(\omega)$ becomes frequency independent at high frequencies. This general tendency of dielectric loss of all the samples can be associated with the prediction that the samples have high and low resistivity for the low and high frequency regions, respectively [42].

On the other hand, the dielectric loss of both pure PPy and PT is very high and VBR doping significantly increases the dielectric loss of both polymers. In this respect, the composites with the highest dissipation factor (i.e. dielectric loss) $(\mathrm{PPy}+(50 \%) \mathrm{VBR}$ and $\mathrm{PT}+(10 \%) \mathrm{VBR}$ composites) may be suitable for decoupling capacitor applications [37].

\subsection{AC electrical conductivity of CPs/VBR composites}

To analyze the AC electrical conductivity of CPs/VBR composites, alternative current (AC) conductivity, $\sigma_{\mathrm{ac}}$, has been calculated by equation 2 [43-45]:

$$
\sigma_{a c}=\omega \varepsilon_{0} \varepsilon^{\prime \prime}
$$

where $\varepsilon_{0}$ represents dielectric constant of free space. According to Jonscher's "universal dielectric response", AC conductivity can be defined as:

$$
\sigma_{a c}=A \omega^{s}
$$

where $\mathrm{A}$ is a frequency independent constant and s is frequency exponent [43]. In accordance with Jonscher's power law, s takes values between zero and one. Jonscher universal power law enables one to determine the conductivity process of materials based on different mechanisms depending on frequency exponent value. While DC conductivity corresponds to $\mathrm{s} \approx 0 ; \mathrm{s} \approx 0.5$ and $0.7 \leqslant \mathrm{~s}<1$ conditions indicate correlated barrier hoping $(\mathrm{CBH})$ and quantum mechanical tunneling (QMT) model, respectively [46-48]. In this context, to determine the $\mathrm{s}$ parameters of the samples, $\ln \sigma_{\mathrm{ac}}=\mathrm{f}(\ln \omega)$ 

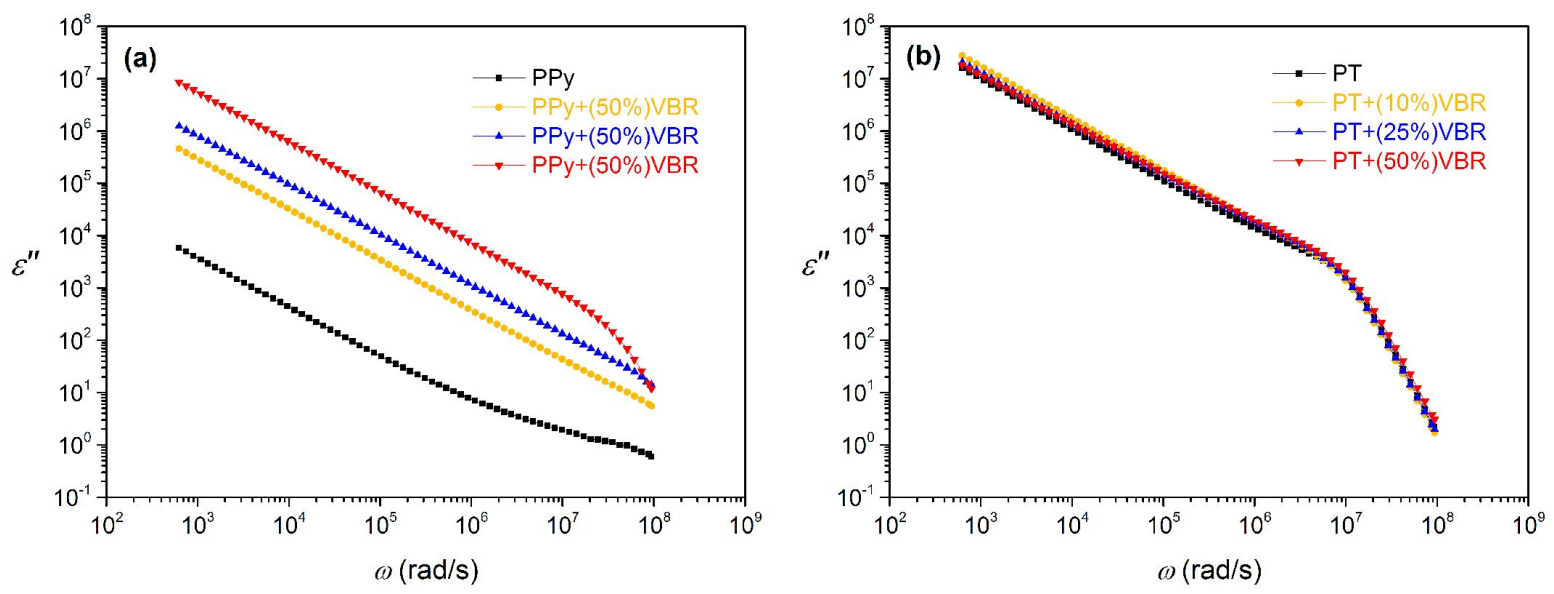

Fig. 6. Angular frequency dependence of $\varepsilon^{\prime \prime}$ for (a) pure PPy and PPy/VBR composites, (b) pure PT and PT/VBR composites.

graphs given in Fig. 7 have been investigated. The slopes of $\ln \sigma_{\mathrm{ac}}=\mathrm{f}(\ln \omega)$ curves (i.e. the value of frequency exponent) have been calculated for different frequency regions shown in Fig. 7 and listed in Table 4.

According to frequency exponent values at low frequency given in Table 4, both pure PPy and PT and their VBR composites exhibit frequency independent, i.e. DC conductivity. As the frequency is increased to high frequency region, it has been determined that while pure PPy exhibits CBH mechanism, pure PT shows DC conductivity at room temperature. It has been also recognized that while increasing VBR doping concentration reduces the $\mathrm{s}$ parameter values for PPy composites, the doping above $25 \%$ VBR increases the s parameter values of PT composites. On the other hand, DC conductivities at $\mathrm{f}=24 \mathrm{~Hz}$ (or $\ln \omega=5$ ) have also been determined by linear exploration method and given in Table 4. In this respect, it has been indicated that VBR doping also increases conductivities at low frequency. For this reason, it has been suggested that the PPy+(50 \%) VBR and PT+(10\%) VBR composites can be utilized as antistatic media and in electromagnetic interference shielding applications due to their high $\sigma_{\mathrm{ac}}$ values and very weak frequency dependence of $\sigma_{\mathrm{ac}}$ in low and high frequency regions [37].

\subsection{Impedance properties}

The electrical behavior of CPs and their VBR composites have been investigated by complex impedance spectroscopy (CIS) measurements.

The complex impedance, $\mathrm{Z}^{*}$ and its real and imaginary components are defined by equation 4 , equation 5 and equation 6 :

$$
\begin{aligned}
& Z^{*}=Z^{\prime}-j Z^{\prime \prime} \\
& Z^{\prime}=\left|Z^{*}\right| \cos \theta \\
& Z^{\prime \prime}=\left|Z^{*}\right| \sin \theta
\end{aligned}
$$

where $\mathrm{j}$ represents $\sqrt{-1}$. To determine the device equivalence of the samples, Nyquist plots (i.e. $-Z^{\prime \prime}=f\left(Z^{\prime}\right)$ ) of the samples have been drawn. The Nyquist plots and equivalent circuits have been given in Fig. 8 and Fig. 9 for PPy and PT samples, respectively.

As shown in Fig. 8 and Fig. 9, except for $10 \%$ VBR doped PPy, all Nyquist plots have a tendency of becoming a semi-circle at high frequency region and a line with various slopes at low frequency region. Moreover, non-Debye type of relaxation, which was firstly predicted by determination of non-zero absorption coefficients, also confirmed 
Table 4. s parameter values of pure PPy and PT samples, PPy/VBR and PT/VBR composites.

\begin{tabular}{lccc}
\hline Sample & s (low frequency) & s (high frequency) & $\begin{array}{c}\sigma_{\mathrm{dc}} \text { [S/cm] (linear } \\
\text { extrapolation) }\end{array}$ \\
\hline \hline PPy & $0.07645 \pm 2.94 \times 10^{-3}$ & $0.58594 \pm 1.44 \times 10^{-2}$ & $1.929 \times 10^{-6}$ \\
PPy $+(10 \%) \mathrm{VBR}$ & $0.03442 \pm 5.17 \times 10^{-4}$ & $0.06364 \pm 2.34 \times 10^{-3}$ & $2.063 \times 10^{-4}$ \\
$\mathrm{PPy}+(25 \%) \mathrm{VBR}$ & $0.05448 \pm 1.57 \times 10^{-3}$ & $0.06091 \pm 1.52 \times 10^{-3}$ & $3.882 \times 10^{-4}$ \\
$\mathrm{PPy}+(50 \%) \mathrm{VBR}$ & $0.04421 \pm 1.13 \times 10^{-3}$ & $0.02641 \pm 1.28 \times 10^{-3}$ & $3.211 \times 10^{-3}$ \\
$\mathrm{PT}$ & $0.02708 \pm 4.06 \times 10^{-3}$ & $0.15479 \pm 4.06 \times 10^{-3}$ & $8.217 \times 10^{-3}$ \\
$\mathrm{PT}+(10 \%) \mathrm{VBR}$ & $0.00804 \pm 4.67 \times 10^{-4}$ & $0.05763 \pm 1.77 \times 10^{-3}$ & $1.565 \times 10^{-2}$ \\
$\mathrm{PT}+(25 \%) \mathrm{VBR}$ & $0.03324 \pm 1.35 \times 10^{-3}$ & $0.12409 \pm 2.14 \times 10^{-3}$ & $1.039 \times 10^{-2}$ \\
$\mathrm{PT}+(50 \%) \mathrm{VBR}$ & $0.06314 \pm 6.96 \times 10^{-4}$ & $0.13116 \pm 1.63 \times 10^{-3}$ & - \\
\hline
\end{tabular}
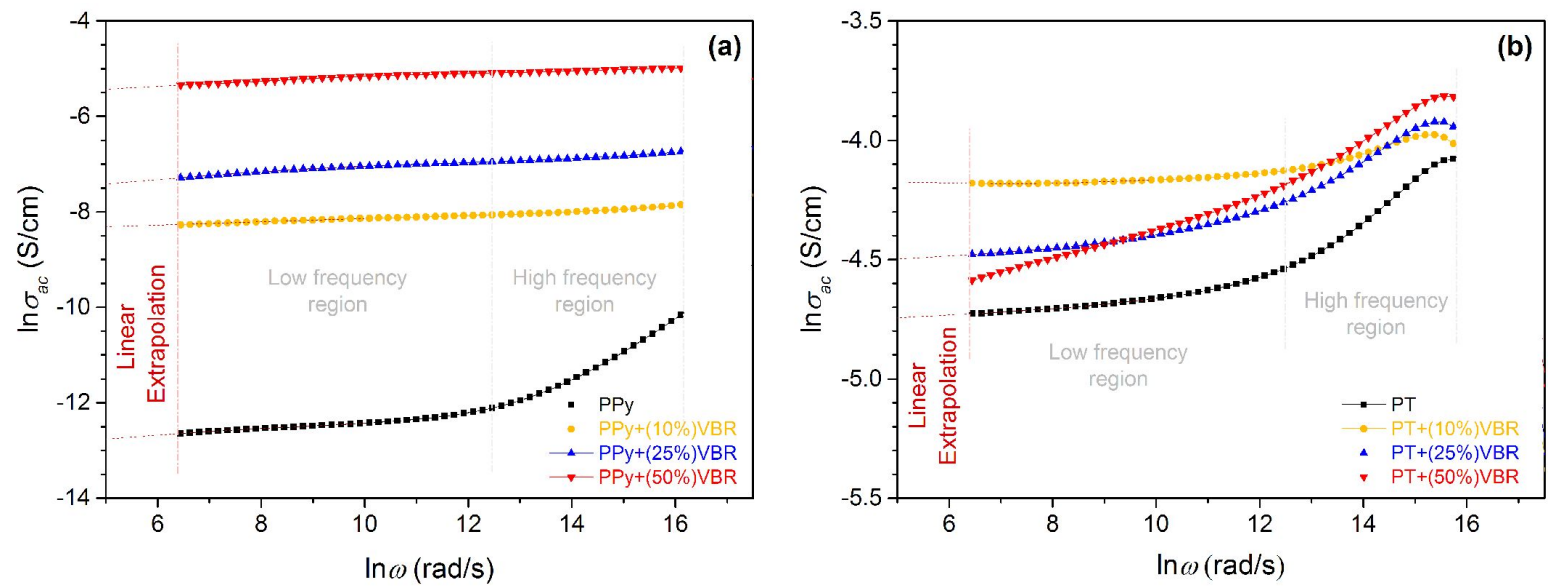

Fig. 7. $\ln \sigma_{\mathrm{ac}}=\mathrm{f}(\ln \omega)$ curves for (a) pure PPy and PPy/VBR composites, (b) pure PT and PT/VBR composites.

itself as semicircles whose centers were below the real axis. The Nyquist plots have also been fitted to determine the equivalent circuits of the composites. Except for $25 \%$ and $50 \%$ VBR doped composites, the samples can be represented by a parallel connected grain resistance $R_{g}$ and grain capacitor $C_{g}$ in series with contact resistance $\mathrm{R}_{\mathrm{s}}$. But according to the fitted curves in Fig. 8c and Fig. 8d, the relatively small semi-circle that appeared at high frequencies indicates grain effects, while the relatively larger semicircle observed at low frequencies refers to the grain boundary effects.

The intercepts of the small and large semicircles on $\mathrm{Z}^{\prime}$ axis correspond to the resistance of $\mathrm{R}_{\mathrm{g}}$ and $\mathrm{R}_{\mathrm{gb}}$, respectively. The related $\mathrm{C}_{\mathrm{g}}$ and $\mathrm{C}_{\mathrm{gb}}$ capacitances have also been calculated by $2 \pi \mathrm{f}_{\mathrm{r}} \mathrm{RC}=1 \mathrm{re}$ lation, where $f_{r}$ is relaxation frequency at which the maximum $Z^{\prime \prime}$ is observed. The related parameters obtained from the Nyquist plots have been listed for each sample in Table 5.

As is seen from Table $5, R_{g b}$ resistance values are higher than the related $\mathrm{R}_{\mathrm{g}}$ values which indicates that the semicircle of grain is masked in a limited frequency range. The higher boundary resistance values may be explained by the fact that the atomic arrangement near the grain boundary is disordered, which results in trapping electrons in the grain boundaries. In addition, while VBR doping considerably decreases the grain resistance of PPy, it is not effective for PT.

\section{Conclusions}

In this work, the influence of VBR doping on dielectric and impedance properties of PPy and PT have been investigated by impedance 
Table 5. The extracted parameters of the circuit elements of pure CPs and CPs/VBR composites.

\begin{tabular}{lccccccc}
\hline \multicolumn{1}{c}{ Sample } & $\mathrm{R}_{\mathrm{s}}[\Omega]$ & $\mathrm{f}_{\mathrm{g}}[\mathrm{Hz}]$ & $\mathrm{f}_{\mathrm{gb}}[\mathrm{Hz}]$ & $\mathrm{R}_{\mathrm{g}}[\Omega]$ & $\mathrm{R}_{\mathrm{gb}}[\Omega]$ & $\mathrm{C}_{\mathrm{g}}[\mathrm{F}]$ & $\mathrm{C}_{\mathrm{gb}}[\mathrm{F}]$ \\
\hline \hline PPy & 0 & 70880 & - & $4.448 \times 10^{4}$ & - & $5.045 \times 10^{-11}$ & - \\
$\mathrm{PPy}+(10 \%) \mathrm{VBR}$ & 188.636 & $6.761 \times 10^{6}$ & - & 631.351 & - & $3.728 \times 10^{-11}$ & - \\
$\mathrm{PPy}+(25 \%) \mathrm{VBR}$ & 158.820 & $1.310 \times 10^{6}$ & 120 & 207.196 & 396.645 & $5.864 \times 10^{-10}$ & $3.344 \times 10^{-6}$ \\
$\mathrm{PPy}+(50 \%) \mathrm{VBR}$ & 28.000 & 85056 & 515.98 & 30.502 & 40.514 & $6.134 \times 10^{-8}$ & $7.613 \times 10^{-6}$ \\
$\mathrm{PT}$ & 7.452 & 85056 & - & 12.585 & - & $1.486 \times 10^{-7}$ & - \\
$\mathrm{PT}+(10 \%) \mathrm{VBR}$ & 6.742 & 49222 & - & 7.665 & - & $4.218 \times 10^{-7}$ & - \\
$\mathrm{PT}+(25 \%) \mathrm{VBR}$ & 6.849 & 59067 & - & 10.436 & - & $2.582 \times 10^{-7}$ & - \\
$\mathrm{PT}+(50 \%) \mathrm{VBR}$ & 8.265 & 59067 & - & 13.000 & - & $2.073 \times 10^{-7}$ & - \\
\hline
\end{tabular}
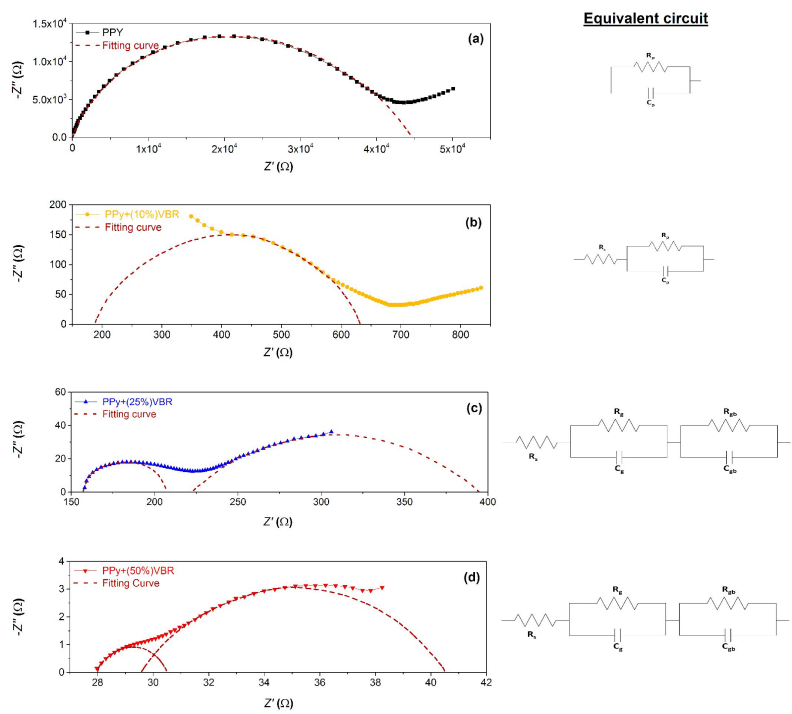

Fig. 8. Nyquist plots, fitted curves and equivalent circuits of (a) pure PPy, (b) PPy+(10\%) VBR, (c) $\mathrm{PPy}+(25 \%) \mathrm{VBR}$, and (d) PPy $+(50 \%)$ VBR.

spectroscopy measurements within the frequency interval of $100 \mathrm{~Hz}$ to $15 \mathrm{MHz}$. Firstly, PPy and PT were synthesized and then the structural characterization was performed by SEM and FT-IR measurements. The VBR which was collected from Van-Turkey, was chosen as a dopant material. The chemical composition and particle size distribution of VBR were determined by XRF and Mastersizer measurements, respectively.

The variations of the real and imaginary components of complex dielectric function of the samples with frequency have been investigated. It was observed that the maximum dielectric

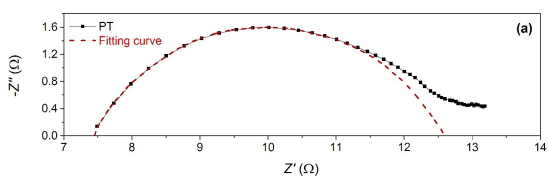

Equivalent circuit
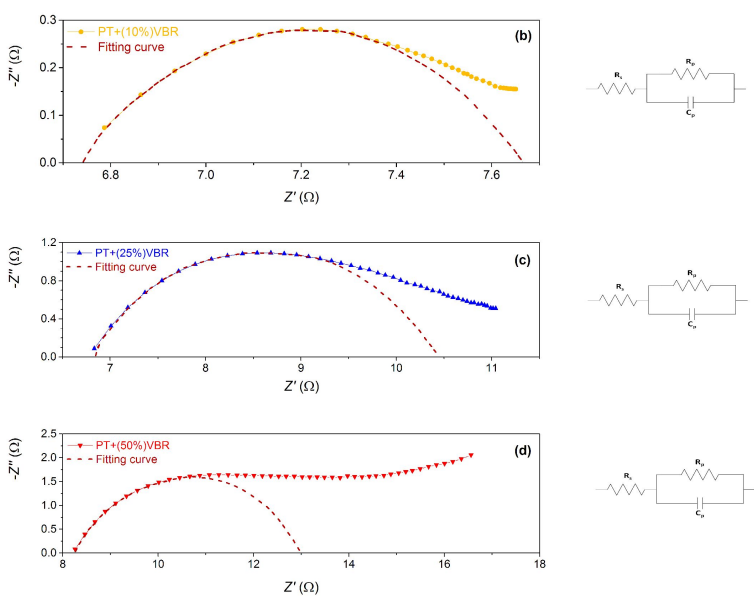

Fig. 9. The Nyquist plots, fitted curves and equivalent circuits of (a) pure PT, (b) PT $+(10 \%) \mathrm{VBR}$, (c) $\mathrm{PT}+(25 \%) \mathrm{VBR}$, and (d) PT $+(50 \%) \mathrm{VBR}$.

permittivity value has been achieved for the maximum VBR doping concentration $(50 \%)$ for both polymers. In addition, dielectric relaxation type of the CPs/VBR composites was investigated. Absorption coefficient, relaxation time constant and dielectric strength have been calculated by application of Cole-Cole relation to $\varepsilon^{\prime}=\mathrm{f}(\omega)$ curves. Due to the non-zero value of absorption coefficient, relaxation mechanism of the samples has been determined as non-Debye type. It was also realized that dielectric strength values considerably 
increase with increasing VBR concentration for both CPs. From this point of view, it is clear that VBR doping makes the dipole orientation easier relative to nondoped samples and reduces the energy requirement of this process. The frequency dependence of conductivity of the samples was investigated using $\ln \sigma_{\mathrm{ac}}=\mathrm{f}(\ln \omega)$ curves by calculating frequency exponent values for the low and high frequency regions. By referring to the $\mathrm{s}$ parameter values, the low and high frequency conductivities have been found to be DC except for pure PPy. Moreover, it has been determined that VBR doping increases the low frequency conductivity.

The impedance spectra of the samples have been studied by Nyquist plots. According to Nyquist plots, it has been realized that VBR doping is mainly effective on the low frequency impedance response of both PPy and PT composites and manifests itself as a decreasing resistivity in the low frequency region.

The imaginary part of dielectric constant (dielectric loss) values of these composites are very high, especially at low frequency region. Consequently, due to high dielectric loss values, high $\sigma_{\mathrm{ac}}$ values and very weak frequency dependence of $\sigma_{\mathrm{ac}}$ in the low and high frequency regions, $\mathrm{PPy}+(50 \%)$ VBR and PT $+(10 \%)$ VBR composites may be utilized in electromagnetic interference shielding applications for the low frequency domain.

\section{Acknowledgements}

This work has been supported by the Yildız Technical University Scientific Research Projects Coordination Department under the Project Number: 2015-01-01-GEP03.

\section{References}

[1] Ates M., Mat. Sci. Eng. C-Mater., 33 (2013), 1853.

[2] Snook G.A., Kao P., Best A.S., J. Power Sources, 196 (2011), 1.

[3] Das T.K., Prusty S., Polym.-Plast. Technol., 51 (2012), 1487.

[4] Long Y-Z., Li M-M., Gu C., WAN M., DuvaIL J-L., LiU Z., Fan Z., Prog. Polym. Sci., 36 (2011), 1415.

[5] Balint R., Cassidy N.J., Cartmell S.H., Acta Biomater, 10 (2014), 2341.

[6] Yakuphanoglu F., Yahia I., Senkal B., SAKr G., FAROOQ W., Synthetic Met., 161 (2011), 817.
[7] Bai Y-M., QIU P., Wen Z-L., Han S-C., J. Alloy. Compd., 508 (2010), 1.

[8] Dhibar S., Bhattacharya P., Hatui G., Das C., J. Alloy. Compd., 625 (2015), 64.

[9] Ke R., Zhang X., Wang L., Zhang C., Zhang S., Mao C., Niu H., Song J., Jin B., Tian Y., J. Alloy. Compd., 622 (2015), 1027.

[10] Li Y., Chen G., Li Q., QiU G., LiU X., J. Alloy. Compd., 509 (2011), 4104.

[11] Liu P., Huang Y., Wang L., Zhang W., J. Alloy. Compd., 573 (2013), 151.

[12] Shen J., Chen K., Li L., Wang W., Jin Y., J. Alloy. Compd., 615 (2014), 488.

[13] Varshney S., Singh K., Ohlan A., Jain V., Dutta V., Dhawan S., J. Alloy. Compd., 538 (2012), 107.

[14] Wang Q., Zheng L., Chen Y., Fan J., Huang H., Su B., J. Alloy. Compd., 637 (2015), 127.

[15] Azadmanjiri J., Hojati-Talemi P., Simon G., Suzuki K., Selomulya C., Polym. Eng. Sci., 51 (2011), 247.

[16] ERdoĞan M.K., Karakişla M., SAÇAK M., J. Macromol. Sci. A, 49 (2012), 473.

[17] Gupta A., Varshney S., Goyal A., Sambyal P., GuPta B.K., DhaWAN S., Mater. Lett., 158 (2015), 167.

[18] Najar M.H., Majid K., J. Mater. Sci.-Mater. El., 26 (2015), 6458.

[19] Le Maitre R.W., Streckeisen A., Zanettin B., LE BAs M., Bonin B., BATEMAN P., Igneous Rocks: a Classification and Glossary of Terms: Recommendations of the International Union of Geological Sciences Subcommission on the Systematics of Igneous Rocks, Cambridge University Press, New York, 2005.

[20] Çetin A., OKutan M., IÇElli O., Yalçin Z., San S., Kibar R., Pesen E., Vacuum, 97 (2013), 75.

[21] El-Shennawi A.W.A., Mandour M.A., Morsi M.M., Abdel-Hameed S.A., J. Am. Ceram. Soc., 82 (1999), 1181.

[22] Yeşilkaya S., ÖZdemir Z.G., CANLi N.Y., Yildiz A., Içelli O., OKutan M., Compos. Part B-Eng., 95 (2016), 488.

[23] Kang Y-Q., CaO M-S., Yuan J., Zhang L., Wen B., Fang X-Y., J. Alloy. Compd., 495 (2010), 254.

[24] Basavaraja C., Veeranagouda Y., Lee K., Vishnuvardhan T., Pierson R., J. Polym. Res., 17 (2010), 233.

[25] Maity A., Sinha Ray S., Macromol. Rapid Comm., 29 (2008), 1582.

[26] Nicho M., Hu H., Sol. Energ. Mat. Sol. C., 63 (2000), 423.

[27] Gao F., Hou X., Wang A., Chu G., Wu W., Chen J., Zou H., Particuology, 26 (2016), 73.

[28] Karim M.R., Lee C.J., Chowdhury A.S., NAHAR N., LEE M.S., Mater. Lett., 61 (2007), 1688.

[29] Yang C., LiU P., Guo J., Wang Y., Synthetic Met., 160 (2010), 592. 
[30] GöK A., Omastová M., Yavuz A.G., Synthetic Met., 157 (2007), 23.

[31] Karim M.R., Lee C.J., Lee M.S., J. Polym. Sci. Pol. Chem., 44 (2006), 5283.

[32] Lu M-D., YAng S-M., Synthetic Met., 154 (2005), 73.

[33] Udum Y.A., Pekmez K., Yildiz A., Eur. Polym. J., 41 (2005), 1136.

[34] Gnanakan S.R.P., Rajasekhar M., SubramaNIA A., Int. J. Electrochem. Sc., 4 (2009), 1289.

[35] Çinar M., KiliÇ M., Karabul Y., Canli N.Y., AlKaN Ü., OKUTAN M, İÇElli O., J. Nanoelectron. Optoe., 11 (2016), 477.

[36] Hong X., Chung D., Carbon, 91 (2015), 1.

[37] Panwar V., Park J.O., Park S.H., Kumar S., Mehra R., J. Appl. Polym. Sci., 115 (2010), 1306.

[38] Blakemore J.S., Solid State Physics, Cambridge University Press, New York, 1985.

[39] Çavdar Ş., Koralay H., TuĞLuOĞLU N., GÜNEN A., Supercond. Sci. Tech., 18 (2005), 1204.

[40] HaAse W., Wróbel S., Relaxation phenomena: liquid crystals, magnetic systems, polymers, high-Tc superconductors, metallic glasses, Springer, New York, 2003.
[41] Meller A., Gmelin Handbook of Inorganic and Organometallic Chemistry. 4th Supplement Volume $3 a$ : $B$ Boron Compounds, The System Boron-Nitrogen, Springer, Berlin, 1991.

[42] Misirlioglu B., Karaman F., Ugraskan V., Serin M., YAZICI O., J. Optoelectron. Adv. M., 17 (2015), 1874.

[43] Jonscher A., J. Mater. Sci., 16 (1981), 2037.

[44] Migahed M., Ishra M., FAhmy T., Barakat A., $J$. Phys. Chem. Solids, 65 (2004), 1121.

[45] Psarras G., Manolakaki E., Tsangaris G., Compos. Part A-Appl. S., 34 (2003), 1187.

[46] Elliott S., Adv. Phys., 36 (1987), 135.

[47] Ghosh A., Phys. Rev. B, 41 (1990), 1479.

[48] Tiwari T., Srivastava N., Srivastava P., Int. J. Electrochem., 2013, 2013.

Received 2017-07-01

Accepted 2018-10-24 